\title{
El sistema intrauterino liberador de levonorgestrel fue más efectivo que los tratamientos médicos usuales para reducir el efecto del sangrado menstrual intenso sobre la calidad de vida
}

The Levonorgestrel- IUS was more effective than usual medical treatment in reducing the effect of heavy menstrual bleeding on quality of life

\begin{abstract}
Objetivos
Comparar la efectividad entre el SIU-LNG y el tratamiento médico convencional con respecto a la mejoría en la calidad de vida en mujeres con sangrado menstrual intenso (SMI) o menorragia que se presentaron a sus médicos de atención primaria.

Diseño, lugar y pacientes

Ensayo pragmático, multicéntrico y aleatorizado, realizado en 63 centros de Reino Unido, entre febrero de 2005 y julio de 2009. Participaron 571 mujeres entre 25 y 50 años, que concurrieron a médicos de atención primaria por menorragia en al menos tres ciclos menstruales consecutivos. Las pacientes fueron asignadas a las ramas de tratamiento en forma aleatorizada y estratificadas por edad, índice de masa corporal, duración de los síntomas, necesidad de anticoncepción y menorragia sola o acompañada por síntomas.
\end{abstract}

\section{Intervención}

Un grupo de 285 pacientes fue tratado con SIU-LNG y el otro grupo de 286 pacientes recibió tratamiento médico convencional (ácido mefenámico, ácido tranexámico, noretindrona, anticonceptivos orales o medroxiprogesterona). Dentro de este último, para el $75 \%$ (n: 215) la prescripción inicial fue ácido mefenámico, ácido tranexámico o la combinación de ambas. Las opciones de tratamientos fueron elegidas por el médico y la paciente en base a la necesidad de anticoncepción y el deseo de evitar el tratamiento hormonal.

Medición de los resultados principales

Se determinó el grado de afectación de la menorragia en la calidad de vida de las mujeres mediante un cuestionario que utilizo la escala MMAS (Multi-Attribute Scale), Este cuestionario evalúa seis parámetros de la vida cotidiana: dificultades prácti- cas, vida social, salud mental, salud física, trabajo y rutina diaria y vida familiar. Se otorgó un puntaje que iba de 0 (afectación severa) a 100 (sin afectación). Secundariamente se evaluó la calidad de vida relacionada con la salud y la actividad sexual, mediante cuestionarios específicos.

Los puntajes se evaluaron antes de la aleatorización y mediante correo electrónico a los 6, 12 y 24 meses. Se registraron los eventos adversos severos.

\section{Resultados principales}

Todos los parámetros evaluados en la escala MMAS mejoraron significativamente con respecto a los puntajes iniciales a los seis meses en ambos grupos (incremento medio de 32,7 para SIU-LNG y de 21,4 para el tratamiento médico convencional; $\mathrm{p}:<0,001)$. Las mejoras se mantuvieron a los dos años, pero fueron significativamente mayores en el grupo del SIU-LNG (diferencia media de 13,4; 95\% IC, 9,9 a16,9 p<0,001).

Con respecto a la evaluación de calidad de vida, la mejoría fue significativamente mayor para el grupo que utilizo SIU-LNG, excepto para el parámetro de salud mental, en el que no hubo diferencia significativa. Tampoco hubo diferencia significativa en cuanto a la evaluación de la actividad sexual.

A los 2 años, el $64 \%$ de las mujeres continuaba utilizando el SIU-LNG versus el $38 \%$ de las mujeres que recibieron el tratamiento médico convencional $(p<0,001)$.

\section{Conclusión}

Tanto el SIU-LNG como el tratamiento médico convencional redujeron los efectos adversos de la menorragia sobre la vida cotidiana de las mujeres, pero el primero fue el método más efectivo.

Fuentes de financiamiento: RNIHR Health Technology Assessment Programme.

\section{Comentario}

La menorragia es un motivo de consulta frecuente que plantea dudas acerca de la terapéutica ideal.

Este ensayo al ser aleatorizado, multicéntrico, con un número de pacientes adecuado y un seguimiento a lo largo de dos años aporta una valiosa herramienta para ser aplicada a la práctica cotidiana.

Es interesante la selección de las pacientes y la evaluación a través de la encuesta acerca de cómo las mujeres creen que el sangrado menstrual intenso interfiere en su vida diaria y no a través de la medición del volumen de sangre perdido en cada ciclo $^{1,2}$, que es un método difícil de aplicar. En definitiva, la afectación en la calidad de vida es lo que lleva a realizar la consulta médica para obtener una solución adecuada.

Si bien en el ensayo, en el $75 \%$ de las pacientes, la prescripción inicial fue ácido tranexámico solo o combinado con ácido mefenámico, en Argentina la experiencia del primero para SMI es escasa y el ácido mefenámico se utiliza en mayor medida para tratar la dismenorrea. De acuerdo a una encuesta realizada en EE.UU, los ginecólogos utilizaron como primera elección los anticonceptivos orales combinados para el SMl ${ }^{3}$.

Existe evidencia de la utilidad del SIU-LNG para la reducción del volumen de sangre en comparación con el tratamiento médico convencional ${ }^{4}$. En Reino Unido, las guías clínicas también lo recomiendan como primera opción ${ }^{5}$.

\section{Conclusiones del comentador}

Si bien los tratamientos médicos convencionales tienen lugar en la terapéutica actual del SMI, la evidencia sugiere que el SIU-LNG constituye una excelente opción para la reducción en el volumen de sangre y la mejoría en la calidad de vida en mujeres con SMI.

Dra. Natalia Andrea Nicolaevsky [ IServicio de Ginecología del Hospital Italiano De Buenos Aires.natalia.nicolaevsky@ hospitalitaliano.org.ar ]

Nicolaevsky N. El sistema intrauterino liberador de levonorgestrel (SIU-LNG) fue más efectivo que los tratamientos médicos usuales para reducir el efecto del sangrado menstrual intenso sobre la calidad de vida. Evid Act Pract Ambul Oct-Dic 2013; 16(4):128. Comentado de: ECLIPSE Trial Collaborative Group. Levonorgestrel intrauterine system versus medical therapy for menorrhagia. N Engl J Med. 2013; 10;368(2):128-37. PMID 23301731.

Referencias

1. Prentice A. Fortnightly review. Medical management of menorrhagia. BMJ. 1999; 319:1343-5.

2. Warner PE, et al. Menorrhagia I: measured blood loss, clinical features, and outcome in women with heavy periods: a survey with follow-up data. Am J of Obstet Gynecol. 2004; 190:1216-23.

3. Matteson KA, et al. Practice pattems and attitudes about treating abnormal uterine bleeding: a national survey of obstetricians and gynecologists. Am J Obstet Gynecol. 2011; 205(4):321. Epub 2011 May 14.

4. Matteson KA, et al; Society of Gynecologic Surgeons Systematic Review Group. Nonsurgical management of heavy menstrual bleeding: a systematic review. Obstet Gynecol. 2013; 121(3):632-43. 5 Heavy menstrual bleeding. London: Royal College of Obstetricians and Gynaecologists; 2007. National Collaborating Centre for Women's and Children's Health. guideline CG44. 